\title{
Correction to: An efficient implementation of pairing-based cryptography on MSP430 processor
}

\author{
Jihoon Kwon ${ }^{1}$. Seog Chung Seo ${ }^{2}$. \\ Seokhie Hong 1
}

\section{Correction to: J Supercomput (2018) 74:1394-1417 https://doi.org/10.1007/s11227-017-2097-4}

The Acknowledgements section is missing in the original article. The Acknowledgements section is given below.

Acknowledgements This work was supported by Institute for Information \& communications Technology Promotion (IITP) grant funded by the Korea government (MSIT) (No. 2014-6-00910, Study on Security of Cryptographic Software).

The original article can be found online at https://doi.org/10.1007/s11227-017-2097-4.

Seokhie Hong

shhong@korea.ac.kr

Jihoon Kwon

htkwon@korea.ac.kr

Seog Chung Seo

seoseogchung82@gmail.com

1 Center for Information Security Technologies (CIST), Korea University, Seoul, Republic of Korea

2 The Affiliated Institute of ETRI, Daejeon, Republic of Korea 